\section{Fatores associados à subnotificação de casos de tuberculose multirresistente no Estado do Rio de Janeiro, Brasil: relacionamento probabilístico entre sistemas de informação}

\author{
Factors associated with underreporting of cases \\ of multidrug-resistant tuberculosis in the state \\ of Rio de Janeiro, Brazil: probabilistic database \\ linkage
}

\section{Factores asociados a la subnotificación de casos de tuberculosis resistente a múltiples fármacos en el estado de Río de Janeiro, Brasil: relación probabilística entre sistemas de información}

Marcela Lopes Bhering da Silva 1,2 Patrícia Durovini 3

Paulo Mota 3

Afrânio Lineu Kritski 1

\section{Resumo}

Neste estudo, estimou-se a proporção e os fatores associados à subnotificação da tuberculose multirresistente (TB-MDR) no Estado do Rio de Janeiro, Brasil, assim como a proporção de óbitos nesse grupo. Realizou-se um estudo de coorte retrospectiva, utilizando a técnica de relacionamento probabilístico entre sistemas de informação. Os casos com resultado do teste de sensibilidade às drogas (TSA) com padrão TB-MDR registrados no Sistema Gerenciador de Ambiente Laboratorial (GAL), no período 2010 a 2017, foram relacionados com casos notificados no Sistema de Tratamentos Especiais de Tuberculose (SITETB). Regressões logísticas simples e múltipla foram realizadas para estimar os fatores associados à subnotificação. Para verificar o óbito, foi realizada a busca dos casos no Sistema de Informações sobre Mortalidade (SIM) e no portal do Tribunal de Justiça do Estado do Rio de Janeiro. Dos 651 casos TB-MDR no GAL, 165 não haviam sido notificados no SITETB, perfazendo uma subnotificação de 25,4\% na amostra. Entre os casos subnotificados, 61 (37\%) foram encontrados nos registros de óbito. Na análise múltipla, ter o exame solicitado por um hospital $(O R=2,86$; IC95\%: 1,72-4,73) esteve associado à subnotificação. No geral, o tempo médio entre a solicitação do exame e a liberação do resultado foi de 113 dias. Entre os casos notificados, o tempo médio entre a solicitação do exame e o início do tratamento foi de 169 dias. Diante disso, é urgente fortalecer as ações de vigilância epidemiológica na TB-MDR, estabelecer e monitorar núcleos de vigilância hospitalar e as rotinas de notificação de TB nos hospitais, rever etapas operacionais, além de unificar os diversos sistemas de informação tornando-os mais ágeis e integrados.

Tuberculose Resistente a Múltiplos Medicamentos; Sistemas de Informação; Vigilância em Saúde Pública; Monitoramento Epidemiológico

\author{
Correspondência \\ M. L. B. Silva \\ Programa Acadêmico de Tuberculose, Faculdade de Medicina, \\ Universidade Federal do Rio de Janeiro. \\ Rua Prof. Rodolpho Paulo Rocco 255, 6o andar, Rio de Janeiro, \\ RJ 22620-170, Brasil. \\ marcelabhering@yahoo.com.br \\ 1 Faculdade de Medicina, Universidade Federal do Rio de \\ Janeiro, Rio de Janeiro, Brasil. \\ 2 Escola Nacional de Saúde Pública Sergio Arouca, Fundação \\ Oswaldo Cruz, Rio de Janeiro, Brasil. \\ 3 Instituto de Estudos em Saúde Coletiva, Universidade Federal \\ do Rio de Janeiro, Rio de Janeiro, Brasil.
}




\section{Introdução}

A tuberculose (TB) causou cerca de 10 milhões de adoecimentos e 1,2 milhão de óbitos em 2019, representando a principal causa de morte por agente infeccioso no mundo e um desafio aos sistemas de saúde 1.

A situação é agravada pelos casos de tuberculose resistente à rifampicina (TB-RR) e multirresistente (TB-MDR), resistentes à rifampicina e isoniazida, as duas principais drogas utilizadas no tratamento da doença. A resistência extensiva (TB-XDR) é acrescida pela resistência a uma fluoroquinolona e ao menos uma droga injetável de segunda linha (amicacina, canamicina ou capreomicina), e a pré-TB-XDR apresenta resistência a fluoroquinolona ou a um injetável de segunda linha, mas não a ambos ${ }^{2}$. Estes casos necessitam de esquemas mais prolongados, dispendiosos e de menor tolerabilidade 1,3 .

Em 2014, a Organização Mundial da Saúde (OMS) aprovou a Estratégia pelo Fim da Tuberculose (End TB) com o objetivo de eliminar a doença como problema de saúde pública. Para isso, as metas são reduzir em $80 \%$ os casos novos e em $90 \%$ as mortes por TB até 2030 , em comparação a 2015, eliminando também o impacto econômico para as famílias afetadas pela doença 4 .

Para que essas metas sejam alcançadas, a OMS reforçou recomendações feitas desde 2010. Entre elas está a garantia que os governos forneçam acesso universal a testes moleculares para promover o diagnóstico de TB droga sensível e droga resistente (TB-DR) e priorizem o início precoce de tratamento 5 .

Além disso, em 2018, ocorreu a primeira reunião de alto nível da Assembleia Geral das Nações Unidas sobre TB que resultou na Declaração Politica sobre Tuberculose, com metas ambiciosas para acelerar os esforços e o compromisso dos países de acabar com a epidemia de tuberculose globalmente até 20306.

Isso é de elevada importância diante da estimativa da OMS de que apenas 38\% dos casos estimados de TB-MDR e TB-RR foram diagnosticados em 2019, deixando um passivo de pessoas a serem alcançadas pelos sistemas de saúde 1. Por outro lado, são escassos os dados de pacientes com TB-RR/ MDR com diagnóstico laboratorial que não foram inseridos nos sistemas de notificação e, portanto, não receberam tratamento adequado, promovendo a manutenção da transmissão do bacilo resistente e maior chance de morbidade e mortalidade 7 .

Em 2017, o Brasil registrou 1.041 casos de TB-DR, sendo 713 TB-MDR 8. O Estado do Rio de Janeiro, apesar de ser um dos mais desenvolvidos economicamente no país, se destacou por ter fraco desempenho no controle da TB. Em 2018, teve o maior coeficiente de mortalidade do país, 4,3 por 100 mil habitantes 9 e o segundo maior coeficiente de incidência entre os casos novos de TB, 63,3 por 100 mil habitantes 10. Em países de alta carga, são escassos os estudos na literatura indexada que analisam a subnotificação de casos de TB-MDR, sendo este um estudo inédito em nível regional no Rio de Janeiro.

Desse modo, este estudo tem por objetivo estimar a proporção e os fatores associados à subnotificação da TB-MDR no Rio de Janeiro, com base nos dados do Gerenciador de Ambiente Laboratorial (GAL - http://gal.datasus.gov.br/GALL/index.php) e do Sistema de Tratamentos Especiais de Tuberculose (SITETB - http://sitetb.saude.gov.br/), assim como estimar a proporção de óbitos nesse grupo.

\section{Método}

\section{Tipo de estudo}

Estudo de coorte observacional retrospectivo utilizando dados secundários. As coortes foram extraídas do GAL e do SITETB.

\section{Seleção da amostra}

O GAL foi desenvolvido pelo Ministério da Saúde, em 2008, com o objetivo de informatizar a rede laboratorial de saúde pública do país, propiciar controle da qualidade dos resultados dos diagnósticos 
e disponibilizar os dados para as vigilâncias epidemiológicas e ambientais para as esferas municipal, estadual e federal 11,12. Para o estudo, foram selecionados todos os exames de teste de sensibilidade às drogas (TSA) para TB-MDR, com resistência a, pelo menos, rifampicina e isoniazida (RH), solicitados e liberados entre agosto de 2010 e maio de 2017, de pacientes residentes no Estado do Rio de Janeiro.

Em 2000, o Brasil iniciou a vigilância epidemiológica da TB-MDR. Em 2004, o país desenvolveu o sistema de informação da tuberculose multirresistente (sistema TBMR), que foi aprimorado e culminou, em 2013, na implantação de um sistema eletrônico de informações - o SITETB. O SITETB é utilizado para a notificação e acompanhamento dos casos de TB que tenham indicação de tratamentos especiais que impossibilitem o uso do esquema básico. Isso pode ser tanto pela ocorrência de reações adversas, toxicidade, alguma comorbidade ou por resistências às drogas 13,14. Além disso, também é utilizado para notificação e acompanhamento dos casos de micobactérias não causadoras de tuberculose (MNT), assim como para controle e dispensação dos medicamentos utilizados nos tratamentos.

No SITETB, foram obtidos os dados dos casos notificados para TB droga resistente (TB-DR) no Estado do Rio de Janeiro e que iniciaram tratamento entre janeiro de 2008 e dezembro de 2018. O período maior teve como objetivo possibilitar a captação dos casos que eventualmente já tivessem iniciado tratamento anteriormente ou que ainda poderiam iniciar até o final de 2018.

\section{Relacionamento das bases}

O relacionamento teve como objetivo identificar casos de TB-MDR com exame confirmado no GAL que não estavam notificados no SITETB. Como o SITETB e o GAL não possuem variável identificadora única entre elas, realizou-se o método de relacionamento probabilístico de registros, com auxílio do software OpenRecLink versão 3.1 (http://reclink.sourceforge.net/) 15. Esse método utiliza campos comuns com o objetivo de identificar, com probabilidades estabelecidas, se os registros pareados pertencem ao mesmo indivíduo 16 .

Foi utilizada uma rotina de múltiplos relacionamentos utilizando os campos comuns entre as bases de dados. As variáveis utilizadas para blocagem foram: código soundex do primeiro e do último nome do paciente, sexo, soundex do primeiro e último nome da mãe e ano de nascimento. Ao todo, foram utilizadas 10 estratégias de blocagem com diversas reorganizações dessas variáveis com o objetivo de aumentar a sensibilidade do processo. Partiu-se de uma chave de blocagem mais restrita com todos os campos disponíveis até a menos específica. Para cada relacionamento, foram estimados os parâmetros utilizando nome e data de nascimento do paciente e nome da mãe. A revisão manual dos pares ocorreu em todos os passos para garantir a qualidade do processo. Como a base do SITETB continha casos notificados até dezembro de 2018, foi feita, por último, uma revisão manual por meio de consulta na base do SITETB após o ano de 2018, objetivando buscar possíveis casos registrados após esse período e/ou pares não relacionados anteriormente.

A revisão manual foi considerada o padrão ouro para o cálculo de valor preditivo positivo (precision), que avalia, dentre todos os pares que o modelo encontrou, quantos estão corretos. É definido como a razão entre os pares verdadeiros encontrados e a soma entre os pares verdadeiros e falsos. Já a medida de sensibilidade (recall) avalia quantos registros não pareados tinham - um par. É definido como a razão entre pares verdadeiros e a soma dos pares verdadeiros e não pares falsos 17.

Como tanto o SITETB como o GAL podiam conter mais de um registro para o mesmo indivíduo, os relacionamentos foram feitos sem excluir os registros duplicados. Ao final, foram selecionados apenas um registro por paciente utilizando o critério de data de solicitação mais antiga do TSA.

Após essa etapa, foram selecionados, entre os casos pareados, aqueles com padrão de resistência atual de multirresistência, resistência extensiva ou em uso de esquema terapêutico TB-MDR. Esse procedimento teve como objetivo garantir que todos os casos relacionados eram multirresistentes.

Foram considerados casos subnotificados aqueles pacientes com TB-MDR confirmada no GAL que não estavam registrados no SITETB. Para verificar se ocorreu o óbito dos casos subnotificados, foi realizada a busca pelo nome do paciente, nome da mãe e data de nascimento no Sistema de Informações sobre Mortalidade (SIM). Como os pesquisadores tinham acesso ao SIM até dezembro de 2014, a busca dos possíveis óbitos ocorridos a partir de janeiro de 2015 foi realizada também no Portal Extrajudicial de Consultas de Nascimentos e Óbitos do Tribunal de Justiça do Estado do Rio de 
Janeiro (http://www4.tjri.jus.br/SEIDEWEB/default.aspx, acessado em 14/Set/2020). Optou-se por utilizar esse portal por entender que é um site público, de fácil acesso e que pode ser extremamente útil para os profissionais de saúde no trabalho cotidiano.

\section{Métodos estatísticos}

De acordo com a natureza da variável, foram usados número (frequência), média (desvio padrão - DP) ou média (intervalo de 95\% de confiança - IC95\%) para descrever as características dos pacientes no geral e, especificamente, de casos notificados e subnotificados.

Foi calculado o tempo médio (dias) entre solicitação do exame e liberação do resultado para todos os casos e, especificamente, de casos notificados e subnotificados. Também foram calculados os tempos das etapas do processo, desde a data da solicitação até o início do tratamento.

Para os casos notificados no SITETB e que iniciaram o tratamento após a data de solicitação do exame, foi calculado o tempo médio (dias) entre solicitação do exame e o início do tratamento para TB-MDR.

Regressões logísticas simples foram realizadas para avaliar a relação entre a subnotificação e as seguintes covariáveis: sexo, faixa etária, categoria de droga resistência (TB-MDR, pré-TB-XDR ou TB-XDR), região do estado da unidade de saúde solicitante (capital, Baixada Fluminense, outras), tipo de unidade de saúde solicitante (hospital ou outras unidades), região da unidade de saúde (capital ou demais regiões), tipo de unidade de saúde (unidade básica ou hospital) e tempo entre solicitação do exame e liberação do resultado.

Foram estimados os odds ratio (OR) com IC95\% e variáveis com nível de significância $\leq 0.20$ na análise simples foram incluídas no modelo de regressão logística múltipla. O teste de Hosmer-Lemeshow foi utilizado para ajuste do modelo e determinação dos odds ratio ajustado (ORa). Variáveis associadas com resultados com significância $\leq 0,05$ no modelo ajustado foram reportadas. As análises estatísticas foram realizadas com o programa Stata versão 13.1 (https://www.stata.com).

Este estudo foi aprovado pelo Comitê de Ética em Pesquisa do Hospital Universitário Clementino Fraga Filho da Universidade Federal do Rio de Janeiro, sob parecer número 3.373.280 (CAAE: 10126919.2.0000.5257).

\section{Resultados}

Entre agosto de 2010 e maio de 2017, foram analisados 10.604 resultados de exames de TSA liberados no GAL, no Estado do Rio de Janeiro, dos quais $1.047(10,4 \%)$ apresentavam resistência simultânea a rifampicina e isoniazida. No SITETB havia 3.358 casos notificados como TB-DR.

Do total, 879 registros do GAL foram recuperados por todas as etapas do relacionamento (precision $=100 \%$ ). Destes registros, $96,3 \%$ foram encontrados por meio das etapas probabilísticas realizadas, $1,9 \%$ por meio de revisão manual e 1,8\% na consulta na base do SITETB após o ano de 2018, também de forma manual. Ao final, 486 registros não foram recuperados por nenhuma das estratégias utilizadas. Medidas para recall não foram possíveis de serem calculadas, pela ausência de um padrão ouro para estabelecer relacionamentos falso-negativos.

Após a etapa de relacionamento, foi selecionado apenas um exame - o mais antigo - por paciente, perfazendo 651 casos. Entre estes, $165(25,4 \%)$ estavam subnotificados, ou seja, não haviam sido notificados no SITETB. Entre os casos pareados, dois foram notificados como casos de monorresistência e não realizaram tratamento terapêutico para multirresistência, sendo considerados casos subnotificados. Entre os casos subnotificados, 61 (37\%) foram encontrados nos registros de óbito (Figura 1).

$\mathrm{Na}$ Tabela 1 foram descritas as variáveis sociodemográficas e clínicas entre pacientes TB-MDR notificados e não notificados no SITETB.

Do total dos 651 casos, $411(63,1 \%)$ eram do sexo masculino, com média de $40( \pm 15,7)$ anos, e faixa etária predominante de 25 a 44 anos $(41,6 \%)$ em ambos os grupos.

A cidade do Rio de Janeiro foi responsável por 369 (56,7\%) solicitações de TSA, seguida pela região da Baixada Fluminense, com 161 (24,7\%). Entre esses municípios, Duque de Caxias e São João de Meriti foram os municípios da Baixada Fluminense que mais solicitaram exames, $58(8,91 \%)$ e 
Figura 1

Fluxograma do estudo.

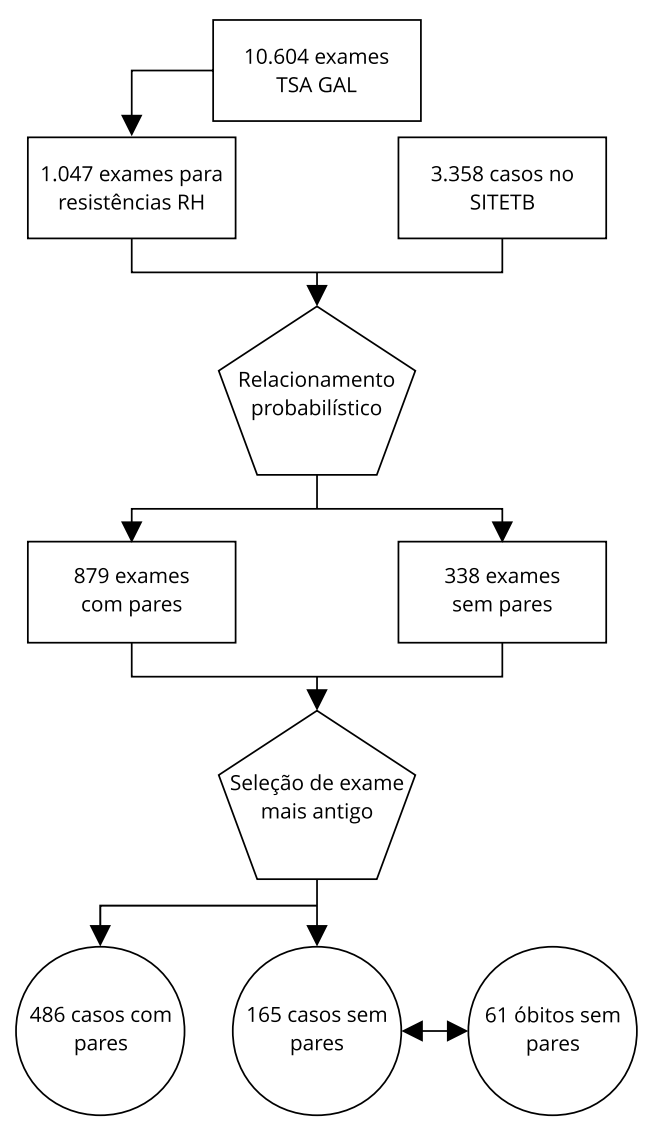

GAL: Sistema Gerenciador de Ambiente Laboratorial; RH: rifampicina e isoniazida; SITETB: Sistema de Tratamentos Especiais de Tuberculose; TSA: teste de sensibilidade às drogas.

34 (5,22\%) e os com mais casos subnotificados, 13 (7,88\%) e 7 (4,24\%), respectivamente. Não houve diferença estatística significante para a subnotificação entre os municípios da Baixada Fluminense $(\mathrm{p}=0,656)$.

As unidades de saúde com maior subnotificação de casos foram o Hospital Municipal Raphael de Paula Souza, com 14 (8,5\%), e o Centro Municipal de Saúde de Duque de Caxias, laboratório central de Belford Roxo e unidade de vigilância sanitária de São João de Meriti, com 5 (3\%) casos cada. Não houve diferença significativa nas proporções da subnotificação entre os anos analisados.

No geral, o tempo médio entre a solicitação do exame e a data de liberação foi de 113 dias (IC95\%: 109-117), variando de 18 a 456 dias (Figura 2). A identificação das espécies e o TSA foram realizados pelo sistema automatizado (BACTEC MGIT 960 - BD Brasil, São Paulo, Brasil) em 529 (81,3\%) dos casos, com tempo médio de 115 dias (IC95\%: 110-119) e pelo método das proporções em 122 (18,7\%), com tempo médio de 107 dias (IC95\%: 99-116) 19. As médias entre os tempos de solicitação do exame pela unidade de saúde e a liberação do resultado no GAL não apresentaram diferença estatística significante $(\mathrm{p}=0,172)$.

O laboratório de referência estadual (Lacen - Laboratório Central de Saúde Pública Noel Nutels) foi o responsável pela realização de 442 (68\%) exames, seguido pelo laboratório do Instituto 
Tabela 1

Características sociodemográficas dos 651 pacientes com teste de sensibilidade às drogas compatível com TB-MDR no Sistema Gerenciador de Ambiente Laboratorial (GAL), notificados e não notificados no Sistema de Tratamentos Especiais de Tuberculose (SITETB).

\begin{tabular}{|c|c|c|c|c|}
\hline Características & $\begin{array}{c}\text { Total de casos } \\
\begin{array}{c}(\%) \\
{[n=651]}\end{array}\end{array}$ & $\begin{array}{c}\text { Notificados } \\
\begin{array}{c}(\%) \\
{[n=486]}\end{array}\end{array}$ & $\begin{array}{c}\text { Não notificados } \\
\begin{array}{c}(\%) \\
{[n=165]}\end{array}\end{array}$ & Valor de $p$ \\
\hline Sexo & & & & 0,554 \\
\hline Feminino & $240(36,9)$ & $176(36,2)$ & $64(38,8)$ & \\
\hline Masculino & $411(63,1)$ & $310(63,8)$ & $101(63,2)$ & \\
\hline Idade [média (DP)] & $40,6( \pm 15,7)$ & $41( \pm 14,7)$ & $40( \pm 18,4)$ & 0,538 \\
\hline \multicolumn{5}{|l|}{ Faixa etária (anos) } \\
\hline $0-24$ & $113(17,4)$ & $77(15,8)$ & $36(21,8)$ & \\
\hline $25-44$ & $271(41,6)$ & $207(42,6)$ & $64(38,8)$ & 0,080 \\
\hline $45-64$ & $229(35,2)$ & $178(36,6)$ & $51(30,9)$ & \\
\hline$\geq 65$ & $38(5,8)$ & $24(4,9)$ & $14(8,48)$ & \\
\hline \multicolumn{5}{|l|}{ Categoria de droga resistência } \\
\hline TB-MDR & $636(97,5)$ & $475(97,7)$ & $160(97,0)$ & \\
\hline Pré-TB-XDR & $10(1,5)$ & $7(1,5)$ & $3(1,8)$ & \\
\hline TB-XDR & $6(1,0)$ & $4(0,8)$ & $2(1,2)$ & 0,850 \\
\hline \multicolumn{5}{|l|}{ Região do Estado } \\
\hline Capital & $369(56,7)$ & $264(54,3)$ & $150(63,6)$ & \\
\hline Baixada Fluminense & $161(24,7)$ & $126(25,9)$ & $35(21,2)$ & \\
\hline Outras & $121(18,6)$ & $96(19,7)$ & $25(15,2)$ & 0,111 \\
\hline \multicolumn{5}{|l|}{ Local } \\
\hline Hospital & $73(11,2)$ & $39(8,0)$ & $34(20,6)$ & \\
\hline Unidade básica & $578(88,8)$ & $447(92,0)$ & $131(79,4)$ & \\
\hline \multicolumn{5}{|l|}{ Tipo de hospital } \\
\hline Municipal & $52(72,2)$ & $30(76,9)$ & $23(67,7)$ & 0,448 \\
\hline Estadual & $3(4,2)$ & $2(5,1)$ & $1(2,9)$ & \\
\hline Federal & $17(23,6)$ & $7(18,0)$ & $10(29,4)$ & \\
\hline \multicolumn{5}{|l|}{ Resistência às drogas [n/N (\%)] } \\
\hline Rifampicina & $651 / 651(100,0)$ & $486 / 486(100,0)$ & $165 / 165(100,0)$ & $<0,001$ \\
\hline Isoniazida & $651 / 651(100,0)$ & $486 / 486(100,0)$ & $165 / 165(100,0)$ & \\
\hline Etambutol & $123 / 651(18,9)$ & $90 / 486(18,5)$ & $33 / 165(20,0)$ & \\
\hline Pirazinamida & $170 / 651(26,1)$ & $140 / 486(28,8)$ & $30 / 165(18,2)$ & \\
\hline Estreptomicina & $138 / 651(21,2)$ & $131 / 486(27,0)$ & $37 / 165(22,4)$ & \\
\hline Capreomicina & $3 / 516(0,5)$ & $3 / 486(0,6)$ & $0 / 30(0,0)$ & \\
\hline Kanamicina & $7 / 9(7,7)$ & $5 / 62(8,1)$ & $2 / 29(6,9)$ & \\
\hline Ofloxacina & $14 / 91(15,4)$ & $10 / 61(16,4)$ & $4 / 30(13,3)$ & \\
\hline Levofloxacina & $2 / 2(100,0)$ & $2 / 2(100,0)$ & 0 & \\
\hline Moxifloxcina & $0 / 2(0,0)$ & $0 / 2(0,0)$ & 0 & \\
\hline $\begin{array}{l}\text { Tempo médio entre solicitação do TSA } \\
\text { e liberação do resultado (dias) }\end{array}$ & $113( \pm 52)$ & $112( \pm 52)$ & $117( \pm 55)$ & 0,492 \\
\hline
\end{tabular}

DP: desvio padrão; TB: tuberculose; TB-MDR: TB multirresistente; TB-XDR: TB extensivamente resistente. 
Média de tempo entre as etapas cadastradas no Sistema Gerenciador de Ambiente Laboratorial (GAL), e o início do tratamento cadastrado no Sistema de Tratamentos Especiais de Tuberculose (SITETB).

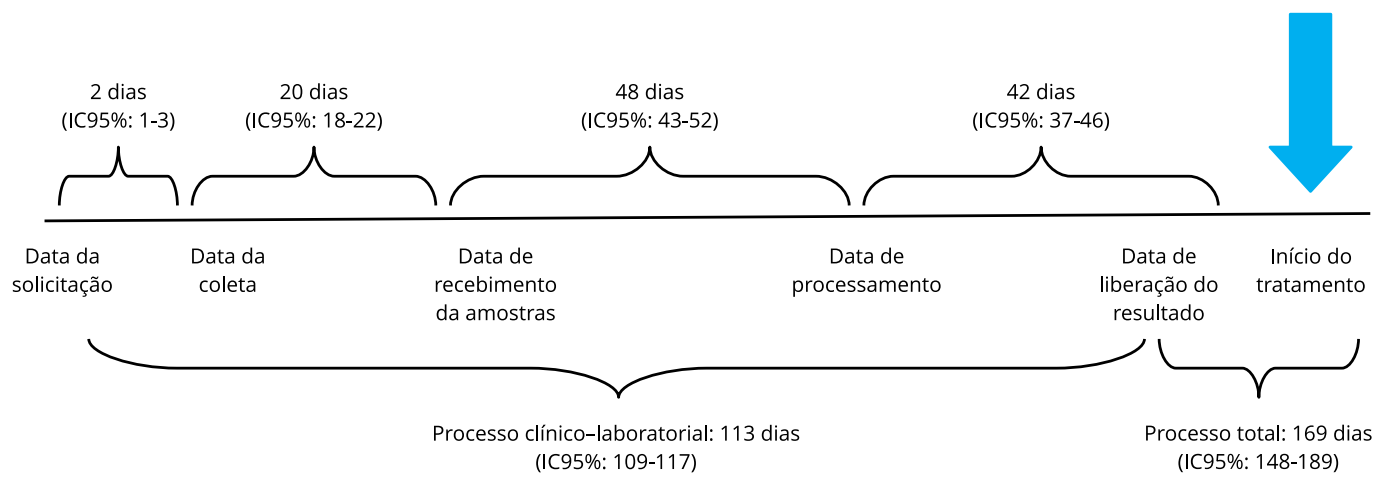

IC95: intervalo de 95\% de confiança.

Nacional de Infectologia Evandro Chagas, Fundação Oswaldo Cruz (INI/Fiocruz), que realizou 141 $(21,7 \%)$ exames.

Na regressão simples, a subnotificação esteve associada com ter o exame solicitado por qualquer unidade de saúde do município do Rio de Janeiro (OR = 1,47; IC95\%: 1,02-2,10) e ter exame solicitado por um hospital de qualquer região do estado ( $\mathrm{OR}=2,97$; IC95\%: 1,80-4,90). Na análise múltipla, a solicitação do exame ter sido realizada por uma unidade hospitalar teve duas vezes $(\mathrm{ORa}=2,86$; IC95\%: 1,72-4,73) mais chances de subnotificação que quando realizado por uma unidade básica (Tabela 2).

Entre os casos que foram notificados no SITETB, o tempo médio entre a data da solicitação do exame e a data do início do tratamento foi de 169 dias (IC95\%: 148-189). Quando o exame foi solicitado no GAL, 41 casos já estavam notificados no SITETB e foram excluídos desse cálculo.

\section{Discussão}

Neste estudo, a proporção de subnotificação de TB-MDR, com base nos dados do GAL e do SITETB no Estado do Rio de Janeiro, foi de 25\%, para o período de 2010 a 2018.

Esse percentual foi similar ao relatado por Bartholomay em estudo que relacionou as bases do GAL e SITETB utilizando casos de TB-DR. Entre 2014 e 2016, em todo o Brasil, o estudo relatou que, entre os 4.054 casos de TB-DR registrados no GAL, $1.626(40,1 \%)$ deles não foram registrados no SITETB, portanto não receberam tratamento para TB resistente. Entre 1.626 pacientes com TB-DR não localizados no SITETB, 42\% eram monorresistentes à isoniazida, 21\% eram multirresistentes e $19,7 \%$ eram polirressistentes. No Estado do Rio de Janeiro, a proporção de subnotificação no SITETB foi de $40 \%$ para os casos TB-DR e de 20,5\% para TB-MDR. O mesmo estudo mostrou que, dos 351 casos de TB-MDR/XDR subnotificados em todo o país, 97 (27,6\%) eram do Estado do Rio de Janeiro 7.

No Brasil, mesmo com o aumento do diagnóstico dos casos de TB-RR, após a implantação do Xpert MTB/RIF, a detecção desses casos não passou de $63 \%$ nos últimos quatro anos 7 . Segundo a OMS, em 2017, o Brasil diagnosticou apenas 55\% dos casos estimados de TB-MDR e TB-RR 19. Isso caracteriza uma elevada proporção de abandono primário (uso de medicamento por menos de 30 dias ou quando o paciente diagnosticado não inicia o tratamento) e, consequentemente, manutenção da transmissão de TB-DR/MDR na comunidade 8 . 
Tabela 2

Fatores preditivos para subnotificação de casos de TB-MDR.

\begin{tabular}{|c|c|c|c|c|}
\hline Preditores & $\begin{array}{c}\text { Simples } \\
\text { OR (IC95\%) }\end{array}$ & Valor de $p$ & $\begin{array}{c}\text { Múltipla } \\
\text { ORa (IC95\%) }\end{array}$ & Valor de $p$ \\
\hline \multicolumn{5}{|l|}{ Sexo } \\
\hline Feminino & 1,00 & & & \\
\hline Masculino & $1,16(0,77-1,60)$ & 0,554 & & \\
\hline \multicolumn{5}{|l|}{ Faixa etária (anos) } \\
\hline $0-24$ & 1,00 & & 1,00 & \\
\hline $25-44$ & $0,66(0,61-1,59)$ & 0,095 & $0,80(0,48-1,34)$ & 0,414 \\
\hline $45-64$ & $0,61(0,37-1,01)$ & 0,057 & $0,74(0,43-1,25)$ & 0,262 \\
\hline$\geq 65$ & $1,24(0,57-2,69)$ & 0,316 & $1,52(0,68-3,37)$ & 0,302 \\
\hline \multicolumn{5}{|l|}{ Categories of drug resistance } \\
\hline TB-MDR & 1,00 & & & \\
\hline Pré-TB-XDR & $1,27(0,32-4,97)$ & 0,729 & & \\
\hline TB-XDR & $1,48(0,26-8,18)$ & 0,650 & & \\
\hline \multicolumn{5}{|l|}{ Região do Estado } \\
\hline Demais regiões & 1,00 & & 1,00 & \\
\hline Capital & $1,47(1,02-2,11)$ & 0,037 & $1,38(0,96-2,01)$ & 0,082 \\
\hline \multicolumn{5}{|l|}{ Tipo de unidade de saúde } \\
\hline Unidade básica & 1,00 & & 1,00 & \\
\hline Hospital & $2,97(1,80-4,90)$ & $<0,001$ & $2,86(1,72-4,73)$ & $<0,001$ \\
\hline Tempo entre solicitação do exame e liberação do resultado & $1,00(0,99-1,00)$ & 0,287 & & \\
\hline
\end{tabular}

IC95\%: intervalo de 95\% de confiança; ORa: odds ratio ajustado; TB: tuberculose; TB-MDR: TB multirresistente; TB-XDR: TB extensivamente resistente.

Outro fator que pode contribuir para a transmissão primária da TB-DR/MDR é o longo tempo observado entre a solicitação do exame pela unidade de saúde e a liberação do resultado pelo laboratório de execução, 113 dias, e entre a solicitação do exame e o início do tratamento, 169 dias. Cabe ressaltar que o exame é solicitado pela unidade de saúde, encaminhado ao laboratório responsável e, posteriormente, para o laboratório de referência para a execução do TSA. Todas essas etapas contribuem para aumento ou diminuição do tempo até o início do tratamento.

Em relação ao método laboratorial utilizado, dados de 2020 da comissão nacional de incorporação de tecnologias no Sistema Único de Saúde (Conitec) mostram que o tempo médio para a detecção de micobactérias utilizando o método automatizado é de 14,07 dias, quase a metade dos 27,08 dias da cultura sólida. Para a realização do TSA, por sua vez, o tempo oscila entre três a 14 dias, pelo sistema automatizado, e de sete a 42 dias, pelo método das proporções 20.

É importante frisar que a maior vantagem da utilização do meio líquido com método automatizado para a detecção da micobactéria e avaliação da sensibilidade aos antimicrobianos é, justamente, o ganho de tempo proporcionado 21. Apesar de o método automatizado ter sido empregado em $81,3 \%$ dos casos estudados, isso não se refletiu em uma maior agilidade na obtenção dos resultados. A linha de cuidados do paciente com TB-DR/MDR é um trajeto que tem início na identificação e confirmação laboratorial das pessoas acometidas, passando pelo acesso ao serviço especializado, até o acompanhamento conjunto na atenção básica. Uma análise dos motivos dos longos intervalos, embora ultrapasse o escopo deste estudo, deve ser realizada para que os ajustes necessários tornem oportuno o cuidado da TB-MDR.

Recentemente, foi descrito que cerca de 30\% dos casos de TB-XDR no Estado do Rio de Janeiro são prováveis casos de infecção primária 22. Além disso, segundo dados do Ministério da Saúde, apenas 56,8\% dos casos de retratamento de TB pulmonar com cultura positiva tiveram o teste de sensibilidade realizado 9 . 
Esse fato sinaliza a urgente necessidade da adoção de medidas efetivas de controle de infecção de TB para diminuir a transmissão. A situação é mais alarmante entre casos com bacilo XDR, que têm proporção de cura em torno de apenas 20\%, pois ainda não há tratamento eficaz para tais pacientes 22 . Revisão de etapas operacionais, como a convocação do paciente para o fornecimento das amostras de escarro, transporte destas entre os laboratórios e agendamento da consulta especializada, poderiam otimizar os processos atualmente adotados com ganho de tempo.

No Brasil, além do GAL, que coleta dados no momento do diagnóstico laboratorial de TB, e do SITETB, que notifica e acompanha casos de TB-DR/MDR ou situações especiais, existem ainda o Sistema de Informação de Agravos de Notificação (SINAN), que reúne informações no início do tratamento da TB ativa e o Sistema de Informação Hospitalar (SIH) 23,24. Entretanto os dados inseridos nesses sistemas não são relacionados entre si, dificultando a vigilância dos casos.

Por outro lado, as unidades de saúde, além da notificação dos casos, investigação de contatos e solicitação dos exames, precisam preencher diversos formulários, como: mapa de medicamentos, ficha de encaminhamento e/ou transferência, boletim de acompanhamento de casos, ficha de solicitação de baciloscopia de escarro, ficha de acompanhamento da tomada diária do medicamento, impresso de resultado PPD, caderneta de controle do tratamento e livro de registro de sintomático respiratório no serviço de saúde 7 .

Isso gera uma sobrecarga de trabalho para os profissionais das unidades de saúde e pode influenciar no acompanhamento dos casos 25 . Além disso, a deficiência do preenchimento das fichas de notificação de tuberculose, precária infraestrutura de informática, qualificação insuficiente de recursos humanos, falta de integração entre profissionais de diferentes setores, fluxo da informação ineficiente entre unidades de saúde e municípios são fatores que limitam o controle da TB 26,27.

$\mathrm{Na}$ ausência de dispositivos que conectem de modo efetivo os diferentes Informações adicionais sistemas de informação relacionados à TB para alertar aos serviços solicitantes sobre a positividade de exames, a qualidade da atenção e a vigilância podem ser afetadas. Nesse período, além da manutenção da transmissão comunitária de formas drogarresistentes de $\mathrm{TB}$, a deterioração clínica dos pacientes pode resultar em aumento das taxas de internação e mortalidade. É provável que esse lapso de informação se reflita nos $37 \%$ de óbitos encontrados entre os casos não notificados. Esse valor é muito superior aos 9\% encontrado em estudo realizado com pacientes TB-MDR de todo Brasil 28 e aos 15,3\% encontrados para pacientes do Estado Rio de Janeiro notificados entre 2000 e 201622 .

Em nosso estudo, o exame ter sido solicitado por uma unidade hospitalar foi o fator associado com a subnotificação. Estudo realizado com pacientes TB sensível apontou que as unidades de pronto-socorro foram importantes na não notificação dos casos. Isso pode ser um indicativo de casos que a vigilância epidemiológica não conseguiu identificar precocemente. $\mathrm{O}$ mais grave é que os casos, embora identificados pelo serviço, não realizaram o tratamento adequado 29.

A subnotificação, além de repercutir na manutenção da cadeia de transmissão da TB-MDR e no aumento da resistência primária, tem como consequência a sobrecarga do sistema de saúde, notadamente a nível hospitalar, ao qual esses pacientes retornarão com agravamento do quadro e menores possibilidades de recuperação.

Quando ocorre falha no sistema básico de saúde, seja pelo acesso difícil para o paciente ou pela baixa resolutividade das equipes, há o aumento dos casos de tuberculose diagnosticados em hospitais, correspondendo geralmente a casos mais graves, com maior tempo de evolução, retardo do diagnóstico e comorbidades 30,31. Apesar das ações prioritárias para o controle da TB se concentrarem nas unidades básicas de saúde e unidades ambulatoriais de referência, torna-se estratégico incluir os hospitais nas ações de controle da TB 32 .

A descentralização do acesso ao SITETB na rede especializada de saúde resultou em maior atenção dos profissionais quanto ao encaminhamento e vinculação do paciente com TB-DR ao tratamento pós-alta hospitalar 13. Porém outros fatores precisam ser considerados para a modificação do quadro encontrado no presente estudo. Pelo observado, torna-se urgente estabelecer e monitorar núcleos de vigilância hospitalar e as rotinas de notificação de TB nos hospitais, com encaminhamento oportuno e responsável do paciente para a atenção básica, com comunicação efetiva entre esses serviços, por meio de e-mail ou telefonema, por exemplo. O uso de documentos em papel, como fichas de referência e contrarreferência, encaminhadas pelos pacientes, pode gerar perda de informação preciosa para a continuidade do cuidado. 
Também é necessário lembrar que as pessoas se movimentam pelos territórios, dentro e entre municípios, e diferentes serviços de saúde podem ser acessados até que elas consigam iniciar o tratamento. Daí a relevância da coordenação do cuidado pela atenção básica junto à atenção especializada e hospitalar 31. A integralidade do cuidado das pessoas com TB depende de estreita articulação entre esses pontos, garantindo não apenas o diagnóstico, mas também a vinculação ao tratamento e a investigação de contatos.

Por isso, a descentralização do acesso ao SITETB e GAL para a rede hospitalar (incluindo os locais de pronto-atendimento), atenção básica e especializada é uma estratégia que poderia ser pensada para aprimorar a comunicação entre os serviços. Assim, pacientes que interromperam um tratamento para TB-DR/MDR poderiam ser identificados mais rapidamente por meio da consulta nesses sistemas, assim que chegassem, por exemplo, em um serviço de saúde de outro município. Da mesma forma, mudanças de regime terapêutico ou resultados de exames laboratoriais realizados na atenção especializada poderiam ser visualizados e acompanhados simultaneamente em todos os níveis de atenção. Essa recomendação está em consonância com o proposto por Ballestero et al. 33 .

A pessoa com TB é única, e a dicotomia entre vigilância e assistência à saúde representa um fator que limita o êxito terapêutico. Sistemas de informação que tenham características de interoperabilidade disponibilizadas aos profissionais de saúde em toda a rede irão contribuir para a vigilância e a comunicação entre especialistas e profissionais da atenção básica, evitando a subnotificação, mortes por TB e a manutenção da transmissão da TB-MDR 34.

Uma limitação do nosso estudo foi não ter conseguido verificar a causa dos óbitos dos pacientes não notificados para avaliar se TB foi uma das causas do óbito. Outra foi não ter mais variáveis clínicas e sociodemográficas dos pacientes para a análise, pois não constam do banco do GAL. Além disso, não foi possível calcularmos medidas de recall (quantidade de pacientes multirresistentes não linkados com o GAL) pela dificuldade de contato com os pacientes devido à escolha de utilização de base de dados secundárias.

Concluímos que, diante desses dados, fica clara a necessidade de fortalecer as ações de vigilância epidemiológica da TB-MDR, especialmente no âmbito hospitalar, estabelecendo e monitorando as rotinas de notificação e o encaminhamento dos pacientes para a atenção básica. É necessário unificar os diversos sistemas existentes, tornando as informações mais ágeis e integradas. Dessa forma será possível realizar o registro, acompanhamento e avaliação dos pacientes e de seus contatos, armazenando desde as informações mais básicas até os dados de triagem, diagnóstico clínico e laboratorial, tratamento e hospitalização.

Por fim, é fundamental estabelecer as causas para reduzir o longo intervalo entre a solicitação dos exames laboratoriais e o início do tratamento da TB-MDR, mitigando a transmissão comunitária, a sobrecarga do sistema de saúde e os óbitos pela doença. Pensando-se na equidade como princípio do SUS, este estudo aponta unidades e municípios que necessitam de um apoio diferenciado e próximo, para a redução da subnotificação, e resultante qualificação do cuidado às pessoas com TB-MDR. 


\section{Colaboradores}

M. L. B. Silva participou da concepção e projeto do estudo, análise e interpretação dos dados, redação do artigo e revisão crítica relevante do conteúdo e aprovação final da versão a ser publicada. P. Durovini participou da redação do artigo, interpretação dos dados, revisão crítica relevante do conteúdo e aprovação final da versão a ser publicada. P. Mota participou da análise e interpretação dos dados e da redação do artigo. A. L. Kritski participou da concepção do estudo e redação do artigo.

\section{Informações adicionais}

ORCID: Marcela Lopes Bhering da Silva (00000002-1568-026X); Patrícia Durovini (0000-00032941-0698); Paulo Mota (0000-0003-1017-1914); Afrânio Lineu Kritski (0000-0002-5900-6007).

\section{Agradecimentos}

Agradecemos à Secretaria Estadual de Saúde do Rio de Janeiro pela disponibilização das bases de dados.

\section{Referências}

1. World Health Organization. Global tuberculosis report 2020. Geneva: World Health Organization; 2020.

2. Banerjee R, Allen J, Westenhouse J, Oh P, Elms W, Desmond E, et al. Extensively drug-resistant tuberculosis in California, 1993-2006. Clin Infect Dis 2008; 47:450-7.

3. Raviglione MC, Smith IM. XDR Tuberculosis - implications for global public health. N Engl J Med 2007; 356:656-9.

4. Uplekar M, Weil D, Lonnroth K, Jaramillo E, Lienhardt C, Dias HM, et al. WHO's new end TB strategy. Lancet 2015; 385:1799-801.

5. World Health Organization. The End TB Strategy: global strategy and targets for tuberculosis prevention care and control after 2015. Geneva: World Health Organization; 2014.

6. Sahu S, Ditiu L, Zumla A. After the UNGA high-level meeting on tuberculosis - what next and how? Lancet Glob Health 2019; 7:e558-e60.

7. Bartholomay P, Pinheiro RS, Johansen FDC, Oliveira SB, Rocha MS, Pelissari DM, et al. Lacunas na vigilância da tuberculose drogarresistente: relacionando sistemas de informação do Brasil. Cad Saúde Pública 2020; 36:e00082219.

8. Ministério da Saúde. Manual de recomendações para o controle da tuberculose no Brasil. 2a Ed. Brasília: Ministério da Saúde; 2019.

9. Departamento de Doenças Condições Crônicas e Infecções Sexualmente Transmissíveis, Secretaria de Vigilância em Saúde, Ministério da Saúde. Tuberculose 2020. Boletim Epidemiológico Especial 2020; (Número especial):1-39.

10. Secretaria de Vigilância em Saúde. Ministério da Saúde. Brasil Livre da Tuberculose: evolução dos cenários epidemiológicos e operacionais da doença. Boletim Epidemiológico 2019; 50:1-18.

11. Jesus R, Bergamo R, Santos LCF, Santos LCF, Matta ASD, Paula Júnior FJ. Sistema Gerenciador de Ambiente Laboratorial: relato de experiência de uma ferramenta transformadora para a gestão laboratorial e vigilância em saúde. Epidemiol Serv Saúde 2013; 22:525-9.

12. Paula FJ, Matta ASD, Jesus R, Guimarães RP, Souza LRO, Brant JL, et al. Sistema Gerenciador de Ambiente Laboratorial - GAL: avaliação de uma ferramenta para a vigilância sentinela de síndrome gripal, Brasil, 2011-2012. Epidemiol Serv Saúde 2017; 26:339-48.

13. Wilhelm D, Rodrigues MV, Nakata PT, Godoy SDC, Blatt CR. Descentralização do acesso ao sistema de informações de tratamentos especiais em tuberculose. Rev Baiana Enferm 2018; 32:e25134.

14. Bartholomay P, Pinheiro RS, Pelissari DM, Arakaki-Sanchez D, Dockhorn F, Rocha JL, et al. Sistema de Informação de Tratamentos Especiais de Tuberculose (SITE-TB): histórico, descrição e perspectivas. Epidemiol Serv Saúde 2019; 28:e2018158. 
15. Camargo Jr. KR, Coeli CM. RecLink 3: nova versão do programa que implementa a técnica de associação probabilística de registros (probabilistic record linkage). Cad Saúde Colet (Rio J.) 2006; 14:399-404.

16. Coeli CM, Camargo Jr. KR. Avaliação de diferentes estratégias de blocagem no relacionamento probabilístico de registros. Rev Bras Epidemiol 2002; 5:185-96.

17. Christen P. Data matching concepts and techniques for record linkage, entity resolution, and duplicate detection. Berlin/New York: Springer; 2012.

18. Departamento de Vigilância Epidemiológica, Secretaria de Vigilância em Saúde, Ministério da Saúde. Manual nacional de vigilância laboratorial da tuberculose e outras micobactérias. Brasília: Ministério da Saúde; 2008.

19. World Health Organization. Global tuberculosis report 2018. Geneva: World Health Organization; 2018.

20. Secretaria de Ciência, Tecnologia, Inovação e Insumos Estratégicos em Saúde, Ministério da Saúde. Relatório de recomendação: cultura líquida automatizada para detecção de micobactérias e teste de sensibilidade aos antimicrobianos utilizados no tratamento da tuberculose. Brasília: Ministério da Saúde; 2020.

21. Said HM, Kock MM, Ismail NA, Baba K, Omar SV, Osman AG, et al. Comparison between the BACTEC MGIT 960 system and the agar proportion method for susceptibility testing of multidrug resistant tuberculosis strains in a high burden setting of South Africa. BMC Infect Dis 2012; 12:369.

22. Bhering M, Duarte R, Kritski A. Predictive factors for unfavourable treatment in MDRTB and XDR-TB patients in Rio de Janeiro State, Brazil, 2000-2016. PLoS One 2019; 14:e0218299.

23. Departamento de Vigilância Epidemiológica, Secretaria de Vigilância em Saúde, Ministério da Saúde. Manual de recomendações para controle da tuberculose no Brasil. Brasília: Ministério da Saúde; 2011.

24. Tourinho BD, Oliveira PB, Silva GDM, Rocha MS, Penna EQAA, Pércio J. Evaluation of the Drug-Resistant Tuberculosis Surveillance System, Brazil, 2013-2017. Epidemiol Serv Saúde 2020; 29:e2019190.

25. Liporaci QFS. Acompanhamento da notificação dos casos de tuberculose pulmonar com confirmação laboratorial no município do Rio de Janeiro. Academus Revista Científica da Saúde 2018; 3:1-6.
26. Almeida Nogueira J, Sá LD, Mello França U, Almeida SA, Lima DS, Figueiredo TMRM, et al. El sistema de información y control de la tuberculosis en municipios prioritarios del estado de Paraíba en Brasil. Rev Esc Enferm USP 2009; 43:119-25.

27. Cunha NV, Cavalcanti MLT, Santos MLF, Araújo VLA, Oliveira e Cruz DM, Pessanha $\mathrm{GF}$, et al. Estrutura, organização e processos de trabalho no controle da tuberculose em municípios do estado do Rio de Janeiro, RJ, Brasil. Interface (Botucatu) 2015; 19:251-63.

28. Bastos ML, Cosme LB, Fregona G, Prado TN, Bertolde AI, Zandonade E, et al. Treatment outcomes of MDR-tuberculosis patients in Brazil: a retrospective cohort analysis. BMC Infect Dis 2017; 17:178.

29. Pinheiro RS, Andrade VL, Oliveira GP. Subnotificação da tuberculose no Sistema de Informação de Agravos de Notificação (SINAN): abandono primário de bacilíferos e captação de casos em outras fontes de informação usando linkage probabilístico. Cad Saúde Pública 2012; 28:1559-68.

30. Ribeiro SA, Matsui TN. Hospitalização por tuberculose em hospital universitário. J Pneumol 2003; 29:9-14.

31. Prado Junior JC, Virgílio TC, Medronho RA. Comparação da proporção de cura por tuberculose segundo cobertura e tempo de implantação de Saúde da Família e fatores socioeconômicos e demográficos no município do Rio de Janeiro, Brasil, em 2012. Ciênc Saúde Colet 2016; 21:1491-8.

32. Spagnolo LML, Tomberg JO, Vieira DA, Gonzales RIC. Detecção da tuberculose: fluxo dos sintomáticos respiratórios e resultados alcançados. Rev Bras Enferm 2018; 71:2543-51.

33. Ballestero JGA, Garcia JM, Bollela VR, Ruffino-Netto A, Dalcolmo MMP, Moncaio ACS, et al. Manejo da tuberculose multirresistente: elementos centrais das recomendações brasileiras. J Bras Pneumol 2020; 46:e20190290.

34. Pellison FC, Rijo RPCL, Lima VC, Crepaldi NY, Bernardi FA, Galliez RM, et al. Data integration in the Brazilian Public Health System for Tuberculosis: use of the semantic web to establish interoperability. JMIR Med Inform 2020; 8:e17176. 


\section{Abstract}

This study estimated the proportion of underreporting of multidrug-resistant tuberculosis (MDR-TB) and associated factors in the State of Rio de Janeiro, Brazil, as well as the proportion of deaths in this group. A retrospective cohort study was conducted using probabilistic database linkage. Cases with the results of the drug sensitivity test (DST) with $M D R-T B$ pattern recorded in the Laboratory Environment Management System (GAL) from 2010 to 2017 were linked to cases reported to the Special TB Treatments System (SITETB). Simple and multiple logistic regressions were performed to estimate factors associated with underreporting. Death was verified by search for cases in the Mortality Information System (SIM) and in the portal of the Rio de Janeiro State Court of Justice. Of the 651 cases of MDR-TB in the GAL, 165 had not been reported to the SITETB, meaning an underreporting rate of $25.4 \%$ in the sample. Among the unreported cases, 61 (37\%) were identified in the death records. In the multiple analysis, the fact that the test was ordered by a hospital $(\mathrm{OR}=2.86$; 95\% CI: 1.72-4.73) was associated with underreporting. Overall, the mean turnaround time between ordering the test and releasing the result was 113 days. Among reported cases, the mean time between ordering the test and initiating treatment was 169 days. The results underline the urgent need to strengthen epidemiological surveillance activities for MDR-TB, establish and monitor hospital surveillance centers and routine TB reporting in hospitals, review operational stages, and integrate various information systems to make them more agile and integrated.

Multidrug-Resistant Tuberculosis; Informations Systems; Public Health Surveillance; Epidemiological Monitoring

\section{Resumen}

En este estudio se estimó la proporción y los factores asociados a la subnotificación de la tuberculosis resistente a múltiples fármacos (TB-MDR) en el Estado de Río de Janeiro, Brasil, así como la proporción de óbitos en ese grupo. Se realizó un estudio de cohorte retrospectiva, utilizando la técnica de relación probabilística entre sistemas de información. Los casos con resultado del test de sensibilidad a las drogas (TSA) con patrón TB$M D R$, registrados en el Sistema Gerenciador de Ambiente Laboratorial (GAL), en el período 2010 a 2017, se relacionaron con casos notificados en el Sistema de Tratamientos Especiales de Tuberculosis (SITETB). Se realizaron regresiones logísticas simples y múltiples para estimar los factores asociados a la subnotificación. Para verificar el óbito, se realizó la búsqueda de los casos en el Sistema de Información sobre Mortalidad (SIM) y en el portal del Tribunal de Justicia del Estado de Río de Janeiro. De los 651 casos TB-MDR en el GAL, 165 no habian sido notificados en el SITETB, lo que equivale a una subnotificación de un 25,4\% en la muestra. Entre los casos subnotificados, 61 (37\%) se encontraron en los registros de óbito. En el análisis múltiple, que el examen haya sido solicitado por un hospital (OR = 2,86; IC95\%: 1,72-4,73) estuvo asociado a la subnotificación. En general, el tiempo medio entre la solicitud del examen y la llegada del resultado fue de 113 dias. Entre los casos notificados, el tiempo medio entre la solicitud del examen y el inicio del tratamiento fue de 169 dias. Ante esto, es urgente fortalecer las acciones de vigilancia epidemiológica en la TB-MDR, establecer y supervisar núcleos de vigilancia hospitalaria y las rutinas de notificación de TB en los hospitales, revisar etapas operacionales, además de unificar los diversos sistemas de información haciéndolos más ágiles e integrados.

Tuberculosis Resistente a Múltiples

Medicamentos; Sistemas de Información;

Vigilancia en Salud Pública; Monitoreo

Epidemiológico
Recebido em 09/Out/2020

Versão final reapresentada 12/Jan/2021

Aprovado em 29/Jan/2021 